\title{
Preparation technology and photoluminescence properties of CdTe nanocrystals in colloidal solutions and polymeric matrices
}

\author{
S.M. Kalytchuk ${ }^{1}$, D.V. Korbutyak ${ }^{1}$, L.P. Scherbak ${ }^{2}$ \\ ${ }^{1} V$. Lashkaryov Institute of Semiconductor Physics, NAS of Ukraine, \\ 45, prospect Nauky, 03028, Kyiv, Ukraine \\ Phone: +38 (044) 525-63-91, fax: +38 (044) 525-63-91,e-mail:div47@isp.kiev.ua \\ ${ }^{2}$ Yuriy Fedkovych Chernivtsi National University \\ 2, Kotsubynsky str., 58012 Chernivtsi, Ukraine \\ Phone: +38 (0372) 526-235, fax: +38 (0372) 552-914, e-mail: l.shcherbak@chnu.edu.ua
}

\begin{abstract}
An analysis of physical mechanisms responsible for the influence of stabilizer type and ambient material on CdTe nanocrystal growth rate and passivation effectiveness of surface dangling bonds has been carried out. The possibility to change properties of nanocrystals by changing the stabilizer type has been shown.
\end{abstract}

Keywords: CdTe nanocrystal, chemical synthesis, optical properties, thioglycolic acid, L-cysteine.

Manuscript received 18.03.09; accepted for publication 14.05.09; published online 30.06.09.

\section{Introduction}

The discovery of new physical phenomena in quantum structures has given rise to the development of novel high-tech devices based on them. On a lot of parameters, such as operation speed, high stability, low energy consumption, etc., they are far superior to their threedimensional analogs. The use of nanocrystals (NCs) based on semiconductor materials II-VI in highly efficient light-emitting devices is considered to be most promising. The basic advantages of these devices are: relatively low cost of the technology employing techniques of colloidal chemistry, which is used to produce NCs based on II-VI materials; high efficiency of emission; relative simplicity of white light-emitting diode fabrication; low threshold of laser emission appearance, etc. [1-6]. The search of optimal technological regimes to be used for the fabrication of light-emitting devices based on NCs of II-VI materials with the preset performance, the choice of matrix where $\mathrm{NCs}$ are incorporated require a detailed investigation of $\mathrm{NC}$ emission characteristics in dependence on technological conditions of growth, NC sizes, passivator type, physicochemical properties of the matrix, etc.

In this work, we have investigated peculiarities of photoluminescence (PL) and optical absorption spectra in CdTe NCs synthesized at various technological regimes in aqueous colloidal solutions as well as in those transferred after synthesis into a polymeric matrix of poly(dialdiaminammonium chloride) (PDDA).

\section{Synthesis of CdTe nanocrystals}

The following reagents were used for the synthesis of CdTe NCs: $\mathrm{CdI}_{2}$ (chemically pure), thioglycolic acid 98+\% (Aldrich), L-cysteine 99.2+\% ("Sfera Sem"), $0.1 \mathrm{M} \mathrm{NaOH}$ (pure), $0.1 \mathrm{M}$ solution of $\mathrm{HCl}$ (pure), tellurium of the brand TB-4, deionized water with the specific resistance of $18 \mathrm{MOhm}$.

The synthesis of CdTe NCs is based on the interaction of ions $\mathrm{Cd}^{2+}$ with $\mathrm{Te}^{2-}$ in an alkaline medium in the presence of passivators, such as thioglycolic acid (TGA), L-cysteine (L-cys) or other mercapto-derivatives [7]. In this case, water solution of ions $\mathrm{Cd}^{2+}$ is prepared by dissolving saline $\mathrm{Cd}\left(\mathrm{ClO}_{4}\right)_{2} \cdot 6 \mathrm{H}_{2} \mathrm{O}$ in deionized water, this saline being characterized by a monoclinic type of the elementary cell with branchy hydrogen bonds [8] While mercapto-derivatives are dissolved in water, the displacement of water molecules, which are in an octahedric environment of $\mathrm{Cd}^{2+}$ ions, takes place (Fig. 1). The source of tellurium anions was telluriumhydrogen $\mathrm{H}_{2} \mathrm{Te}$, that was obtained electrochemically, and argon was used as an inert atmosphere. When $\mathrm{H}_{2} \mathrm{Te}$ gas passes through the solution, a spontaneous formation of CdTe NCs of nanometer sizes takes place. The size of NCs can be controlled by changing the concentration of 
$\mathrm{Cd}^{2+}$ or mercapto-derivatives, $\mathrm{pH}$ of the solution or the reaction duration. Sulphur atoms that are included in the mercapto-derivatives form the bonds with the surface cadmium atoms of CdTe NCs. This prevents conglutination of $\mathrm{NCs}$, improves their size dispersion. The growth of CdTe NCs as a result of chemical reactions in an aqueous solution can be described by a three-stage process (see for example $[9,10]$ ). At the first stage, a formation of micronuclei (clusters) of CdTe from ions $\mathrm{Cd}^{2+}$ and $\mathrm{Te}^{2-}$ occurs in oversaturated solution. At the second stage, cluster sizes rise while the degree of solution oversaturation decreases. These two processes are associated with the ejection of water and mercaptoderivative molecules and are determined by the oversaturated solution free energy, similar to the mechanism proposed for the synthesis of $\mathrm{SiO}_{2}$ nanoparticles in colloidal solutions [11]. During these stages, CdTe clusters are formed up to a critical size with a strongly developed surface $[9,10,12]$. At the third stage, when a critical size is reached, cluster sizes grow by a diffusive mass transfer from smaller clusters to greater ones (dissolution of smaller clusters in favour of greater ones) according to the Lifshits-Sliozov mechanism [13], that is stimulated by reduction in the free surface energy of NC.

To increase stability, the solutions were subjected to thermal treatment in a boiling water bath for 1 hour. To perform layers of CdTe QDs in a polymeric matrix, a glass substrate, colloidal solution of CdTe NCs, and $2 \%$ solution of PDDA with $\mathrm{pH}=6.4$ were used. The fabrication of CdTe NC films in a polymeric matrix was carried out by successive immersing the glass substrate into the solution of PDDA for $5 \mathrm{~min}$, rinsing in deionized water for $3 \mathrm{~min}$, and storing in a synthesized NC solution for $10 \mathrm{~min}$ with subsequent rinsing in deionized water for $3 \mathrm{~min}$. After this, the cycle was repeated to deposit 30 layers.

\section{Experimental results}

The excitation of PL was carried out using the $\mathrm{He}-\mathrm{Cd}$ laser with the wavelength $325.0 \mathrm{~nm}$ and power $10 \mathrm{~mW}$. The PL spectra were registered with a setup based on the spectrometer MDR-23 equipped with a cooled photomultiplier FEU-62 or FEU-100. The temperature during PL measurements was varied from 4.2 to $300 \mathrm{~K}$.

Fig. 1 shows typical spectra of optical absorption (right) and PL (left) for the synthesized CdTe NCs with different percent relation of stabilizers TGA and L-cys. A PL spectrum is composed of a relatively narrow line caused by the recombination of excitons in the NC [14]. The energy position of the PL exciton line maximum at room temperature for various samples was within the range of 2.1-2.4 eV. We have estimated the mean size of NC basing on a simplified model assuming that the Gaussian size distribution of NCs is realized, the band of absorption maximum position being determined by the $\mathrm{NC}$ mean radius, and its half-width characterizes the dispersion of the NC sizes [15]. As a result, mean values

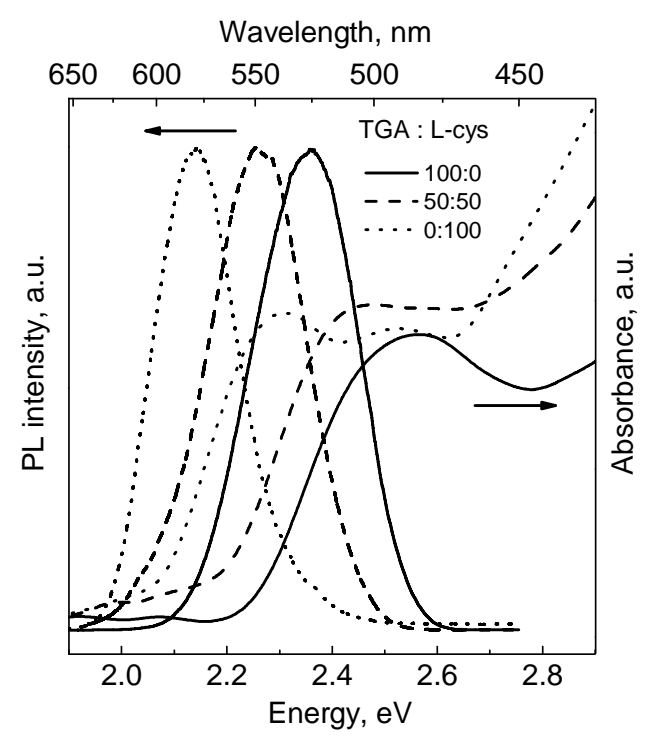

Fig. 1. PL and absorption spectra for CdTe NCs in dependence on the relation of molar stabilizer concentrations TGA:L-cys.

for the NC diameters are obtained for the following relations TGA:L-cys: $1.8 \mathrm{~nm}$ - for TGA:L-cys $=100: 1$, $2.4 \mathrm{~nm}$ - for TGA:L-cys $=50: 50$, and $3.1 \mathrm{~nm}-$ for TGA:L-cys $=0: 100$. They are in agreement with the dependence of the band of absorption energy maximum on the NC size given in [16].

It is found out that an increase in the relation of molar concentrations L-cys / TGA at the synthesis of $\mathrm{NCs}$ gives rise to a long-wave shift of the maxima in the spectra of optical absorption and the maximum of the PL exciton band (from $2.36 \mathrm{eV}$ - for TGA-passivator to $2.14 \mathrm{eV}$ - for L-cys-passivator) and a reduction in its half-width (by $40 \mathrm{meV}$ ). This phenomenon is observed because L-cys forms weaker bonds Cd-S than TGA, since the L-cys molecule is geometrically much longer (more solid), and respectively, the binding energy of the L-cys molecule with an $\mathrm{NC}$ is lower than that of TGA molecule.

The mechanism mentioned is proved by the results on thermal treatment at $100{ }^{\circ} \mathrm{C}$ for 1,2 , and 3 hours of NCs with different relations TGA:L-cys (Fig. 2). When TGA prevailed, then under thermal treatment the PL line was somewhat broadened and shifted towards the longwave region, but much slower than in the case when Lcys dominated. As the main process during the $\mathrm{NC}$ growth is the Oswald ripening, we can state that by passivation of NCs, L-cys forms weaker bonds at the surface than TGA. This promotes the Oswald ripening, i.e. NC size increasing.

In this work, to reveal the recombination mechanism of nonequilibrium charge carriers in CdTe NCs, we studied the temperature dependence of the maximum energy position and the integral intensity of $\mathrm{PL}$ in nanocrystals. Substantial differences were found in the temperature dependences of emission spectral parameters 


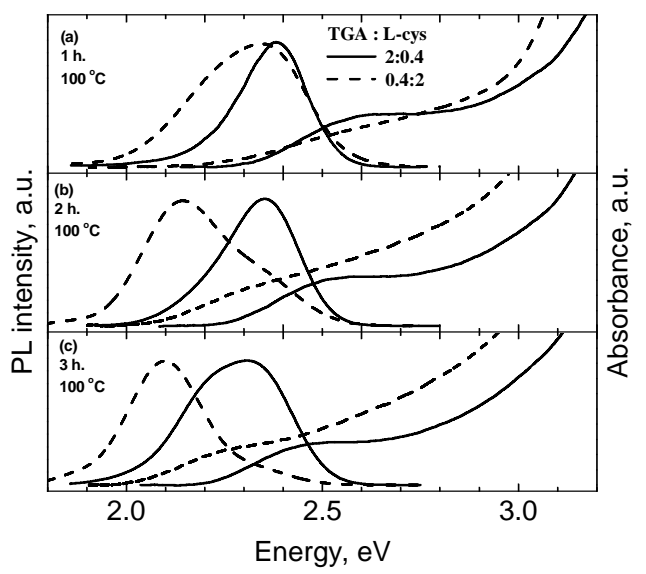

Fig. 2. PL and absorption spectra of CdTe NCs thermally treated at $100{ }^{\circ} \mathrm{C}$ during 1,2 , and 3 hours.

of CdTe NCs depending on the stabilizer type (Fig. 3). In the region up to $150 \mathrm{~K}$, the PL intensity does not change in the case of passivation with TGA. At the temperatures above $150 \mathrm{~K}$, the intensity begins to descend, which is typical for NCs. In the case of passivation with L-cys, the PL intensity decreases beginning with $20 \mathrm{~K}$ that is accounted for by a lower efficiency of passivation with Lcys compared to that of TGA.

One of the reasons for such a PL dependence, to our mind, is that the passivation efficiency in the case of TGA is greater than that of L-cys. The analysis of the experimental data obtained has been carried out within the framework of the model, which takes into account charge carrier recombination with the participation of traps for electrons and holes. The values for the energy of thermally-activated nonradiative recombination and the energies of trap levels have been obtained. Noteworthy is that the exciton PL intensity, when the temperature increases from 4.2 to $300 \mathrm{~K}$, decreases only by a factor of 8 . This fact proves high temperature stability of emission properties of NCs.

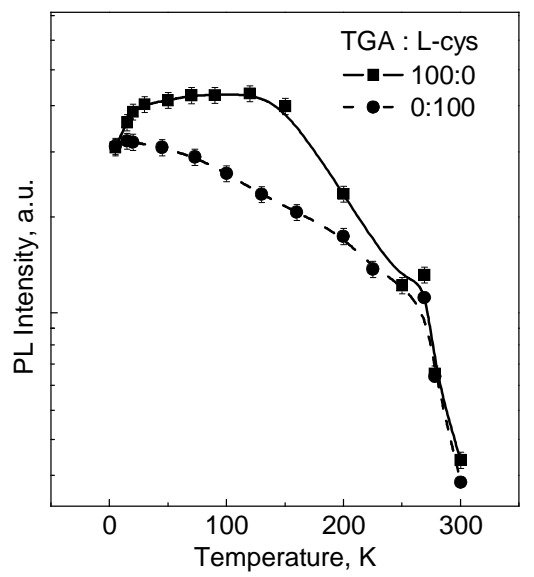

Fig. 3. Temperature dependence of the PL integral intensity for the samples with different relations of passivators TGA:L-cys.

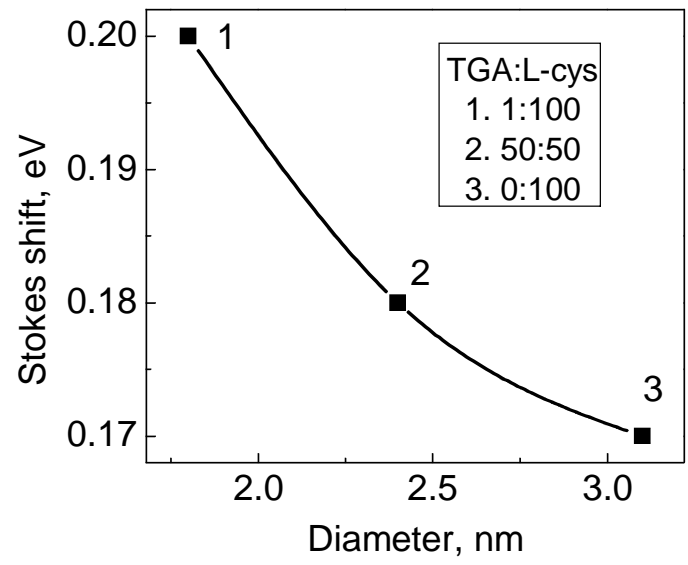

Fig. 4. The Stokes shift dependence on CdTe NC sizes.

From the PL and optical absorption spectra of CdTe NCs, we have determined the Stokes shift value in dependence on NC sizes (Fig. 4). With NC size decreasing from 3.1 to $1.8 \mathrm{~nm}$, the Stokes shift increases from 170 to $200 \mathrm{meV}$ accounted for by an increase in the electron-phonon coupling (Table). The Huang-Rhys factor characterizing the degree of the electron-phonon coupling is found from the formula $\Delta E_{s t}=2 S \cdot \hbar \omega_{\mathrm{LO}}$. Thus, as the NC size decreases, the degree of the electron-phonon coupling increases.

Table. The Huang-Rhys factor dependence on CdTe NC sizes.

\begin{tabular}{|c|c|c|c|}
\hline$N_{s}$ & $\Delta E_{s t}, \mathrm{eV}$ & $d, \mathrm{~nm}$ & Huang-Rhys factor, $S$ \\
\hline 1 & 0.200 & 1.8 & 4.7 \\
\hline 2 & 0.180 & 2.4 & 4.2 \\
\hline 3 & 0.170 & 3.1 & 4.0 \\
\hline
\end{tabular}

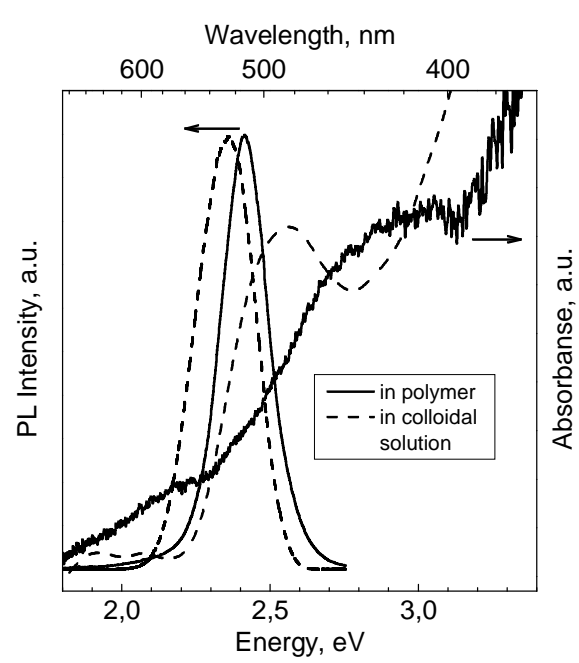

Fig. 5. The PL and absorption spectra of CdTe NCs: solid line - in a polymer, dashed line - in a colloidal solution. 
Fig. 5 shows the absorption spectra for CdTe NCs transferred into a polymeric matrix PDDA in comparison with the initial colloidal solution of $\mathrm{CdTe}$ NCs.

By comparing the PL and absorption spectra with those for colloidal NCs, one can see that the energy position of the PL maximum does not practically change, and the PL line half-width for NCs in polymeric matrices is somewhat narrower; this proves a smaller scatter of sizes for NCs. As to the Stokes shift, when transferring $\mathrm{NCs}$ from a colloidal solution into a polymeric matrix, it increases from 230 to $610 \mathrm{meV}$. A detailed calculation of the matrix influence on the Stokes shift value will be carried out in the process of further investigations.

\section{Conclusions}

In this work, the CdTe NCs have been grown, which were passivated with thioglycolic acid and L-cysteine, and their luminescent properties have been studied.

The observed peculiarities in the PL and optical absorption spectra as well as in the Stokes shift are explained by the influence of the passivator nature (structure) on CdTe NC growth and of the ambient polarization on the excitonic transfers in the NCs studied.

\section{Acknowledgements}

This work was supported in part by State Fundamental Researches Foundation of Ukraine, Russian-Ukrainian Program "Nanophysics and nanoelectronics" and Program "Nanostructured systems, nanomaterials and nanotechnologies" NAS of Ukraine.

\section{References}

1. D.L. Klein, R. Roth, A.K.L. Lim, A.P. Alivisatos, and P.L. McEuen // Nature 389, p. 699 (1997).
2. B.A. Ridley, B. Nivi, and J.M. Jacobson // Science 286, p. 746-749 (1999).

3. V. Colvin, M. Schlamp, and A.P. Alivisatos // Nature 370, p. 354 (1994).

4. W.U. Huynh, J.J. Dittmer, A.P. Alivisatos // Science 295, p. 2425-2427 (2002).

5. R.P. Raffaelle, S.L. Castro, A.F. Hepp, S.G. Bailey // Progress in Photovoltaics: Research and Applications 10, p. 433-439 (2002).

6. V.I. Klimov, A.A. Mikhailovsky, S. Xu, A. Malko, J.A. Hollingsworth, C.A. Leatherdale, H.-J. Eisler, and M.G. Bawendi // Science 290, p. 314 (2000).

7. N. Gaponik, D.V. Talapin, A.L. Rogach, K. Hoppe, E.V. Shevchenko, A. Kornowski, A. Eychmüller, and H. Weller // J. Phys. Chem. B 106, p. 7177 (2002).

8. R. Karlin, Magnetochemistry. Mir, Moscow, 1989 (in Russian).

9. B. Delmon, Kinetics of Heterogeneous Reactions. Mir, Moscow, 1972 (in Russian).

10. A. Sheludko, Colloidal Chemistry. Mir, Moscow, 1984 (in Russian).

11. G.V. Kukolev, Chemistry of Silicium and Physical Chemistry of Silicates. Vysshaya shkola, Moscow, 1966 (in Russian).

12. P. Politi, G. Grenet, A. Marty, A. Ponchet, and J. Villain // Phys. Repts. 324, p. 271 (2000).

13. I.M. Lifshitz and V.V. Slyozov // J. Phys. Chem. Solids 19, p. 35 (1961).

14. S.V. Gaponenko, Optical Properties of Semiconductor Nanocrystals. Cambridge University Press, Cambridge, 1998.

15. H. Matheu, T. Richard, J. Allegre, P. Lefebre, G. Arnaud, and W. Granier // J. Appl. Phys. 77, p. 287 (1995).

16. J. Perez-Conde, A.K. Bhattacharjee, M. Chamarro, P. Lavallard, V.D. Petrikov, and A.A. Lipovskii // Phys. Rev. B 64, 113303 (2001). 